\title{
USO DEL COEFICIIENTE DE CORRELACIÓN Y DESVIACIÓN ESTÁNDAR EN LA SELECCION DE PORTAFOLIOS DE ACTIVOS FINANCIEROS DE RENTA VARIABLE
}

\section{USING THE CORRELATION COEFFICIENT AND STANDARD DEVIATION IN THE SELECTION OF VARIABLE INCOME FINANCIAL ASSET PORTFOLIOS}

\author{
Nicko Alberto Gomero Gonzales \\ Doctor en Ciencias Económicas - Docente Principal de la Facultad de Ciencias Contables - Universidad Nacional \\ Mayor de San Marcos, Lima, Perú. - Email: ngomerog@unmsm.edu.pe (Autor Corresponsal) \\ Víctor Ricardo Masuda Toyofuku \\ Máster en Tributación y Política Fiscal - Docente Asociado de la Facultad de Ciencias Contables - Universidad Nacional \\ Mayor de San Marcos, Lima, Perú. - Email: vmasudat@unmsm.edu.pe \\ Santiago Bazan Castillo \\ Contador Público Colegiado - Docente Asociado de la Facultad de Ciencias Contables - Universidad Nacional \\ Mayor de San Marcos, Lima, Perú. - Email: sbazanc@unmsm.edu.pe
}

[Recibido: 18/05/2016 Aceptado: 16/08/2017]

\section{RESUMEN}

Una característica común de los mercados en donde se negocian activos financieros de renta variable y fija, es su volatilidad. Este escenario de incertidumbre genera oportunidades para acumular riqueza vía estructuración de portafolios óptimos, pero para ello es necesario que el inversionista maneje una serie de herramientas financieras, económicas, matemáticas entre otras, para optar por la mejor posición en este mercado. Por ello para validar los modelos de selección de portafolio se hizo uso de la estadística descriptiva, cuya operacionalización permitió desarrollar carteras de alto y bajo riesgo. El método que se utilizó para el desarrollo del presente trabajo fue de tipo descriptivo, utilizando para ello información histórica de diferentes acciones enlistadas en diferentes índices bursátiles. En el trabajo se llega a demostrar que a través del uso de herramientas estadísticas es posible estructurar portafolios con diferentes grados de riesgo, el cual es medido por la desviación típica, además determinar cómo las acciones reaccionan ante cambios en el mercado bursátil, determinado con ello la Beta de los activos.

\section{PALABRAS CLAVE}

Mercado bursátil, activos, volatilidad, Beta, riesgo, estructuración, portafolios

\begin{abstract}
A common feature of the markets where financial assets are traded in equity and fixed, is its volatility. This scenario of uncertainty generates opportunities to accumulate wealth by structuring optimal portfolios, but for that it is necessary for the investor to manage a series of financial, economic, and mathematical tools, among others, to opt for the best position in this market. Therefore, to validate portfolio selection models, descriptive statistics were used, whose monitoring operation was developed with high and low risk portfolios. The method used for the development of this work was descriptive, using historical information of different actions listed in different stock indices. In the work it is possible to demonstrate that through the use of tools it is possible to structure portfolios with different degrees of risk, which is measured by the standard deviation, in addition to determine how the stock reacts to changes in the stock market, determined with this Beta of the assets.
\end{abstract}

\section{KEYWORDS}

Stock market, assets, volatility, Beta, risk, structuring, portfolios.

\section{INTRODUCCIÓN}

La volatilidad de los activos de renta variable, el cual refleja su nivel de riesgo, exige que los analistas bursátiles o en todo caso los que están ligados a esta industria, manejen una serie de instrumentos estadísticos que les haga posible tomar posiciones favo- rables en este mercado. Más aun, en actuales coyunturas en que los mercados muestran inestabilidad, hace posible que se presenten una serie de problemas a la hora de estructurar portafolios con activos de renta variable. El comportamiento cíclico de estos activos, que los analistas le denominan volatilidad, origina escenarios de incertidumbre que muchas veces conlleva al debilitamiento o mermas de las carteras o portafolios. Claro está que, si la decisión es la correcta, la acumulación de riqueza de quien especula saldrá robustecida con el portafolio estructurado.

Como Citar: Gomero, N., Masuda, V. y Bazan, S. (2017). Uso del coeficiente de correlación y desviación estándar en la selección de portafolios de activos financieros de renta variable. Quipukamayoc, 25(49), 129-140. doi: http://dx.doi.org/10.15381/quipu.v25i49.14288 
En el mundo bursátil existen inversionistas y especuladores. Los primeros proyectan obtener rendimientos a largo plazo, por ello sus portafolios o paquetes de activos están marcados por la cautela o bajo riesgo. Pero los segundos tipos de actores financieros dirigen sus "apuestas" a obtener altos rendimientos en un plazo inmediato; su perfil de tomadores de riesgo les induce a manejar carteras con mucho más volatilidades, que en sí, son los tipos de activos que generan mayores grados de rendimiento. La agresividad de sus portafolios es una característica básica de quienes quieren obtener los máximos rendimientos en el muy corto plazo, el cual es característico de los especuladores. En estos tipos de mercado, apostar por la cautela en el manejo de activos puede conducir a bajos niveles de riesgo, pero a la vez podrían reportar rentabilidades exiguas que no cubrirían las exigencias de este actor bursátil. Como se argumenta, en estos mercados están los adversos y amantes al riesgo, los primeros manejan carteras no agresivas pero seguras, pero los segundos optan por portafolios que les inducen a obtener mayores niveles de ganancia pero asumiendo altos niveles de riesgo.

Bajo este contexto es conveniente señalar a Burton (2012) quien señala lo siguiente:

A estas alturas quizás sea una buena idea explicar lo que quiere decir para mí "invertir", y de qué manera distingo esta actividad de los especuladores considera la inversión una forma de adquirir activos para conseguir unos beneficios en forma de ingresos que se puede predecir de una forma razonable (...) y/o su apreciación a largo plazo a largo plazo. Lo que a menudo diferencia una inversión y la posibilidad de predecir estas ganancias. Un inversor compra acciones que probablemente vayan a producir una rentabilidad y plusvalías seguras en los próximos años y décadas.

El concepto del Burton define claramente lo que es un inversionista y un especulador, pero ambos, participando en el mercado bursátil apuntan a lograr rentabilidades que cubran sus expectativas y compensen los riesgos asumidos.

Dependiendo de la estructuración de sus portafolios que mucho depende en cierta forma de la calidad de los activos, el inversionista o especulador va a lograr las rentabilidades que espera, es así también como lo señala Serrano (2013) quien dice que "el inversor en bolsa obtiene valor si revaloriza su inversión, es decir, si los dividendos que recibe más el aumento de valor de sus acciones superan las pérdidas de valor del dinero debido a la inflación. En caso contrario, pierde valor" (p.20). Claro está que los beneficios que reciba el actor bursátil, materializados en dividendos y ganancias de capital, como se mencionó, va a estar anclada al tipo de activo que incluya en sus portafolio.

El riesgo se va a diversificar en función como se maneje una cartera, en este sentido Serrano (2013) argumenta:

La teoría moderna de carteras (...) y el modelo CAPM de valorización de acciones (...) presentan un concepto fundamental: la diversificación de las inversiones. Para ello, diferencian el riesgo individual o idiosincrático, que es diversificable y por tanto se puede eliminar, del riesgo general o de mercado que no se puede evitar. (p.83)

Detrás de esta apreciación subyace el hecho que una cartera adecuada- mente estructurada para reducir los riesgos no sistémicos implica tomar en cuenta activos inversamente correlacionados, o en todo caso con coeficientes de este tipo que reflejen poca relación de asociatividad o de dependencia.

Marcelo (2015, p.52), explica claramente la importancia del coeficiente de correlación, estadístico que es básico para estructurar portafolios, señalando que expresa la medida de ajuste de $\mathrm{Y}$ como función lineal de $\mathrm{X}$. El autor define matemáticamente este coeficiente de la forma siguiente:

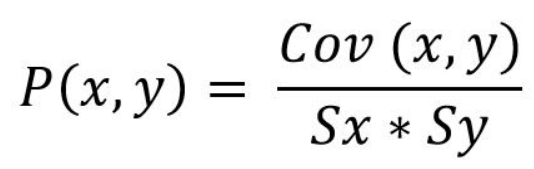

Dónde:

$\mathrm{P}(\mathrm{x} / \mathrm{y})=$ coeficiente de correlación de $\mathrm{x} / \mathrm{y}$

$\operatorname{Cov}(\mathrm{x}, \mathrm{y})=$ covarianza de $\mathrm{x}, \mathrm{y}$

$\mathrm{S} \mathrm{x}=$ desviación típica de $\mathrm{X}$

Sy $=$ desviación típica de $y$

El rango de la correlación va de $(-1$, 1). Si el resultado es negativo indica que entre las variables $\mathrm{x} / \mathrm{y}$ existe una correlación inversa, si es positiva, la relación es directa, si es cero, no existe ningún tipo de relación entre estas variables. Los resultados que se pueda obtener al manejar cartera de activos es muy importante, por ejemplo si un inversionista elige dos activos de renta variable con un coeficiente de correlación negativa y cercana a -1 , estará tomando poco riesgo, pero por el contrario si opta por una combinación de activos con coeficiente positivo y cercano a 1 , el perfil del inversor proyectará una alta preferencia al riesgo. En el primer caso, debido al poco riesgo que se asume se podría obtener mínimas ganancias, pero en el segundo caso, la rentabilidad obte- 
nida podría ser mayor, pero con una elevada propensión al riesgo. Como se puede apreciar, utilizando esta herramienta estadística se puede tomar decisiones en el mercado de valores.

La covarianza y la desviación típica son estadísticos que participan para determinar el coeficiente de correlación. En el primer caso, la covarianza refleja el tipo y grado de asociatividad entre los dos activos que conforman una cartera y por otro lado, la desviación típica expresa el grado de volatilidad de cada activo. Es conveniente precisar, que cuanto mayor sea este coeficiente, la volatilidad o riesgo de los activos será mayor. Por ello se argumenta que si un especulador o inversionista es propenso asumir elevados riesgos con la finalidad de lograr los mayores niveles de rentabilidad, elegirá activos de elevadas varianzas o desviaciones típicas. Si bien, con los dos estadísticos señalados se puede medir el grado de volatilidad del activo, pero el más usado para este fin es el de la desviación típica o estándar. Las formulas se detallan a continuación.

Primero se determina la varianza.

$$
\sigma^{2}=\frac{\sum_{i=1}^{n}\left(x_{i}-\bar{x}\right)^{2}}{n}
$$

Al determinar la raíz cuadrada de este resultado, se determina la desviación típica.

$$
\sigma=\sqrt{\frac{\sum_{i=1}^{n}\left(x_{i}-\bar{x}\right)^{2}}{N}}
$$

En donde:

$\mathrm{Xi}=$ rentabilidad del activo en un periodo determinado

$\mathrm{X}=$ promedio de los rendimientos de los activos

$\mathrm{N}$ = número de observaciones

Una medida más precisa para determinar la volatilidad del activo es el coeficiente de variación (CV), que inclusive permite tomar una mejor

decisión cuando se tienen los mismos resultados de riesgo para los activos o para el portafolio. La fórmula es como sigue:

\section{$C V=\frac{\text { desviación típica de las rentabilidaddes }}{\text { promedio de las rentabilidades }}=\frac{\sigma}{x}$}

Bodie, Kane \& Marcus (2004) señala: Como ahora pretendemos formar una cartera arriesgada de dos activos arriesgados, necesitamos entender cómo interactúan las incertidumbres en la rentabilidad de los activos. Parece ser que el determinante clave del riesgo de la cartera es la medida en que la rentabilidad de los dos activos tiende a variar bien en el mismo sentido, o en forma contraria. Los riesgos de la cartera dependen de la interrelación de las rentabilidades de los activos de la cartera. La apreciación de estos autores nos debe conducir a pensar la importancia que se le debe de dar a las estadísticas o matemáticas para optar por la mejor decisión de inversión en activos financieros. (p.127)

Bajo esta misma línea de análisis, Gitman \& Joehnx (2009), señala:

Con el propósito de lograr sus metas de inversión, integrara una cartera de inversiones apropiadas y usará la diversificación es decir, la inclusión de diversos instrumentos de inversión de una cartera para obtener mayores rendimientos o exponerse a menores riesgo, del que se tendría se limitará sus inversiones a solo unos instrumento. La diversificación es el término financiero que corresponde al antiguo consejo "no pongas todos los huevos en una sola canasta. (p.12)

Diversificar, no solo implica acumular acciones en una cartera, sino la pregunta es ¿qué tipo de acciones? Para responder a esta pregunta en parte, podemos acudir a la estadística y operacionalizar instrumentos que se teorizan en esta disciplina.

Por otro lado, es conveniente señalar lo que expresa Gitman \& Johenk (2009) sobre la forma como seleccionar una cartera y así manejar adecuadamente la relación riesgo rendimiento:

- Con datos de rendimiento históricos o proyectados, calcule el rendimiento esperado durante un determinado periodo de tenencia.

- Con datos de rendimientos históricos o proyectados, evalué el riesgo relacionado con la inversión. La evaluación subjetiva del riesgo, el uso de la desviación estándar o el coeficiente de variación de los rendimientos, así como el uso de medidas más complejas, como el beta $(\ldots)$ son los principales métodos disponibles para los inversionistas individuales

- Evalúe el comportamiento del riesgo rendimiento de cada inversión alternativa para asegurarse que el rendimiento esperado es razonable dado el nivel de riesgo.

- Seleccione el instrumento de inversión que ofrece los rendimientos más altos con relación al nivel de riesgo que se desea asumir.

Como se puede apreciar, también estos autores recomiendan el uso de 
instrumentos estadísticos para la estructuración de portafolios.

Otro indicador básico para tomar decisiones en el mercado bursátil es la Beta de los activos, el cual refleja su grado de volatilidad con respecto a las fluctuaciones del mercado. Según esta teoría, la rentabilidad de las acciones responde a los cambios del mercado, sensibilidad que no es uniforme para cada tipo de activo, por ello un inversionistas puede tomar la decisión de comprar acciones de alto o bajo riesgo o en todo caso estructurar un portafolio con Betas que denoten volatilidades distintas.

Brealey, Myers \& Marcus (2004) indican sobre Beta:

Algunas de las acciones resultan menos afectadas que otras por las fluctuaciones del mercado. Los directores de inversiones hablan de acciones "agresivas" o "defensivas". Las acciones defensivas no son muy sensibles a las fluctuaciones del mercado.

Por el contrario las acciones agresivas amplifican cualquier movimiento del mercado. (...) Las acciones tienen altos Betas mayores a 1, indicando que su rentabilidad tiende a responder en más de una unidad ante los cambios unitarios de rentabilidad del mercado. (p. 225)

Para determinar este indicador existen distintas alternativas estadísticas, siendo una de estas como el que se señala a continuación:

$$
B i=R(i, m) \frac{\sigma_{i}}{\sigma_{m}}
$$

Donde:

$\mathrm{Bi}=$ beta del activo

$\mathrm{R}(\mathrm{i}, \mathrm{m})=$ coeficiente de correlación del activo (i) con respecto al mercado(m) $\sigma_{i}=$ desviación típica del activo $\sigma_{\mathrm{m}}=$ desviación típica del mercado

El otro método matemático es el de la ecuación de regresión lineal simple, en donde la pendiente va a representar a la Beta del activo:

$\mathrm{Y}=\mathrm{Bo}+\mathrm{B} 1(\mathrm{X})$

O en todo caso:

$\mathrm{Ri}=\mathrm{Bo}+\mathrm{B} 1(\mathrm{Rm})$

Dónde:

$\mathrm{Ri}=$ Rendimiento del activo

$\mathrm{RM}=$ rendimiento del mercado

$\mathrm{B} 1=$ Beta del activo

Se realizó un estudio descriptivo, que utiliza información histórica obtenida de las principales índices bursátiles del mundo para probar la importancia de los estadísticos, como el coeficiente de correlación y la desviación típica, para tomar decisiones en este mercado, a través de la estructuración de portafolios óptimos.

Los diferentes grados de volatilidad de los activos que se negocian en los principales mercados bursátiles del mundo, es un hecho normal, claro está que detrás de estas oscilaciones se encuentran una serie de fundamentos internos de las empresas que las emiten, o en todo caso a hechos exógenos, de tipo económico o político, que es imposible diluirlos con la conformación de portafolios, sean o no agresivos.
Las Bolsas Norteamericanas categorizada como la más importante del mundo, en donde encontramos al índice Dow Jones, Nasdaq, S\&P o las Asiáticas, en donde están el Hang Seng, Shangai, entre otros, si bien son unas de las más fuertes del mundo, pero no están libres de esas oscilaciones que son aprovechadas por los especuladores para obtener rentabilidades de corto plazo. Por cierto, también es muy apreciado por inversionistas cautelosos, que más que en un corto plazo, proyectan ganancias en un largo plazo.

Dado estos niveles de incertidumbre en los mercados, en donde se negocian acciones de las empresas más importantes del mundo, como son por ejemplo, Bayer, McDonald, Apple, Caterpillar, Coca Cola, Boing, Repsol, agrupados claro está en distintos Índices Bursátiles, generan un escenario de riesgo en donde es necesario instrumentar una serie de estadísticos, tal como se referencio en el marco teórico, para tomar las mejores decisiones de inversión. Este concepto es válido para la compra de un activo o para la estructuración de portafolios.

En la Tabla 1 se observa la rentabilidad de cuatro acciones que se cotizan en el Dow Jones los cuales presentan diferentes grados de sensibilidad, los cuales quedan evidenciados con la desviación típica, que según los resultados, el activo que presenta mayor variabilidad es el Microsoft, debido que el estadísticos de riesgo llega al $1,28 \%$, seguido de Boing con un $0,94 \%$.

Si un inversionista o especulador quisiera adquirir un activo y evaluar su riesgo podría bastar este estadístico, pero no será de utilidad si el propósito 
es estructurar portafolios. Para este fin sería más conveniente utilizar el confidente de correlación, de esta forma se podría asumir posiciones de alto o bajo riesgo.

Tabla 1.

Rentabilidad de acciones: 2017

Fuente: Índice Bursátil. Dow Jones. www.investing.com - Elaboración propia

\begin{tabular}{ccccc} 
& Coca Cola & MacDonald & Microsoft & Boing \\
\hline 04.12 .2017 & $0,56 \%$ & $-1,29 \%$ & $-3,85 \%$ & $2,40 \%$ \\
\hline 05.12 .2017 & $0,06 \%$ & $1,36 \%$ & $0,63 \%$ & $-0,88 \%$ \\
\hline 06.12 .2017 & $0,41 \%$ & $0,28 \%$ & $1,45 \%$ & $0,99 \%$ \\
\hline 07.12 .2017 & $-1,45 \%$ & $-0,33 \%$ & $-0,35 \%$ & $1,32 \%$ \\
\hline 08.12 .2017 & $-1,03 \%$ & $0,14 \%$ & $2,00 \%$ & $1,38 \%$ \\
\hline 11.12 .2017 & $0,04 \%$ & $0,06 \%$ & $1,26 \%$ & $-0,96 \%$ \\
\hline 12.12 .2017 & $-0,09 \%$ & $-0,59 \%$ & $0,41 \%$ & $2,37 \%$ \\
\hline 13.12 .2017 & $1,34 \%$ & $0,76 \%$ & $-0,27 \%$ & $0,65 \%$ \\
\hline 14.12 .2017 & $0,28 \%$ & $-0,24 \%$ & $-0,78 \%$ & $0,70 \%$ \\
\hline 15.12 .2017 & $0,35 \%$ & $0,53 \%$ & $2,52 \%$ & $0,02 \%$ \\
\hline 18.12 .2017 & $-0,56 \%$ & $0,08 \%$ & $-0,54 \%$ & $0,75 \%$ \\
\hline 19.12 .2017 & $0,43 \%$ & $-0,47 \%$ & $-0,64 \%$ & $0,37 \%$ \\
\hline 20.12 .2017 & $-0,11 \%$ & $-0,71 \%$ & $-0,36 \%$ & $0,22 \%$ \\
\hline 21.12 .2017 & $-1,05 \%$ & $-0,19 \%$ & $-0,02 \%$ & $-0,97 \%$ \\
\hline 22.12 .2017 & $-0,02 \%$ & $-0,25 \%$ & $0,01 \%$ & $0,02 \%$ \\
\hline 26.12 .2017 & $0,48 \%$ & $-0,08 \%$ & $-0,13 \%$ & $0,09 \%$ \\
\hline 27.12 .2017 & $0,26 \%$ & $0,36 \%$ & $0,09 \%$ \\
\hline 28.12 .2017 & $-0,46 \%$ & $0,80 \%$ & $0,01 \%$ & $-0,49 \%$ \\
\hline 29.12 .2017 & $0,35 \%$ & $0,25 \%$ & $\mathbf{0 , 9 4 \%}$
\end{tabular}

En la matriz de correlación se aprecia por un lado acciones que están directamente correlacionados como son Microsoft y McDonald, lo cual significa que las rentabilidades de ambos activos van en la misma dirección, lo cual denota cierto nivel de riesgo para estructurar un portafolio. Si bien, obtener un coeficiente de correlación de $59,88 \%$, proyecta una relación moderada entre estos dos activos, pero el riesgo será mayor si la decisión se inclinara a elegir acciones con correlaciones negativas tal como es el caso de la combinación de Microsoft y Coca Cola cuya correlación es de - 17,32\% o en todo caso entre Boing y McDonald con una correlación de $-45,21 \%$. Si un inversionista bursátil quiere asumir el menor nivel de riesgo o contar con mayor cobertura, debe elegir la combinación de activos que tenga la mayor correlación negativa, si en caso contrario se prefiere el riesgo se optará por una cartera con la mayor correlación positiva. Cuando el coeficiente de correlación se acerca a 1 , el inversionista o especulador pierde cobertura y se expone en mayor grado a los riesgos, el cual debe ser compensado con mayores rentabilidades.
Si bien la matriz de correlación es un instrumento importante para tomar posiciones en el mercado bursátil, pero no es suficiente para optimizar la elección de activos. Para ello es conveniente utilizar otro estadístico denominado desviación típica para el portafolio. Para su determinación un elemento importante es el coeficiente de correlación, por lo que, si la opción es asumir bajos riesgos, o elegir un portafolio con desviaciones típicas que tiendan a disminuir, debe elegir activos con correlaciones negativas y cercanas a -1 , pero si el propósito es asumir altos riesgos o carteras con 
elevadas desviaciones típicas, el inversionistas deberá optar por activos cuyas rentabilidades tengan la misma dirección en su recorrido. El modelo matemático para determinar el riesgo del portafolio es el siguiente:

$$
\sigma_{p}^{2}=W_{x}^{2} \cdot \sigma_{x}^{2}+W_{y}^{2} \cdot \sigma_{y}^{2}+2\left(W_{x} \cdot W_{y} \cdot R_{(x, y)} \cdot \sigma_{x} \cdot \sigma_{y}\right)
$$

Donde:

$\sigma_{\mathrm{p}}{ }^{2}=\quad$ varianza del portafolio

$\stackrel{\mathrm{p}}{\mathrm{W}}(\mathrm{x} / \mathrm{y})=$ participación de cada activo en el porfolio. $\sigma_{\mathrm{x}}=$ desviación típica del activo $\mathrm{X}$

$\sigma_{\mathrm{y}}^{\mathrm{x}}=$ desviación típica del activo $\mathrm{Y}$

$\mathrm{R}(\mathrm{x} / \mathrm{y})=$ correlación del activo $\mathrm{X} \mathrm{y}$ de $\mathrm{Y}$

$S^{2}(p)=$ varianza del portafolio
La desviación típica del portafolio $\sigma(\mathrm{p})$ es la raíz cuadrada de la varianza

$$
\sigma(p)=\sqrt{\sigma_{(p)}^{2}}
$$

Tabla 2.

Matriz de correlaciones

Fuente: Indice Bursátil. Dow Jones. www.investing.com /Elaboración propia

\begin{tabular}{ccccc} 
& Coca Cola & MacDonald & Microsoft & Boing \\
\hline Coca Cola & 1 & & & \\
\hline McDonald & $12,08 \%$ & $100,00 \%$ & $100,00 \%$ & 1 \\
\hline Microsoft & $-17,32 \%$ & $59,88 \%$ & $-35,65 \%$ & 1 \\
\hline Boeing & $-1,91 \%$ & $-45,21 \%$ & & \\
\hline
\end{tabular}

Portafolio 1 - Coca Cola y McDonald

Al analizar el portafolio 1 se observa una correlación del $12,08 \%$ entre

las acciones Coca Cola y McDonald, pero el primero de los activos presenta mayor volatilidad o riesgo por tener mayor desviación típica, el cual llega a $0,65 \%$. Si la cartera es estructurada con un $50 \%$ para cada activo, el riesgo que se obtiene para la cartera es de $0,47 \%$

Tabla 3.

Portafolio de Coca Cola y McDonald

Fuente: www.investing.com / Elaboración propia

\begin{tabular}{ccc} 
& Coca Cola & Mcdonald \\
D.Tipica & $0,65 \%$ & $0,60 \%$ \\
\hline Cartera & $50,00 \%$ & $50,00 \%$ \\
\hline Covarianza & $0,00047 \%$ & \\
\hline C. Correlación & $12,08 \%$ & $0,002 \%$ \\
\hline & VAR & $0,47 \%$ \\
\hline
\end{tabular}

En el Figura 1 se observa la mayor volatilidad de Coca Cola con relación a
McDonald, el cual queda complementado con el resultado estadístico de la desviación típica de $0,65 \%$ y $0,60 \%$ respectivamente para cada activo 


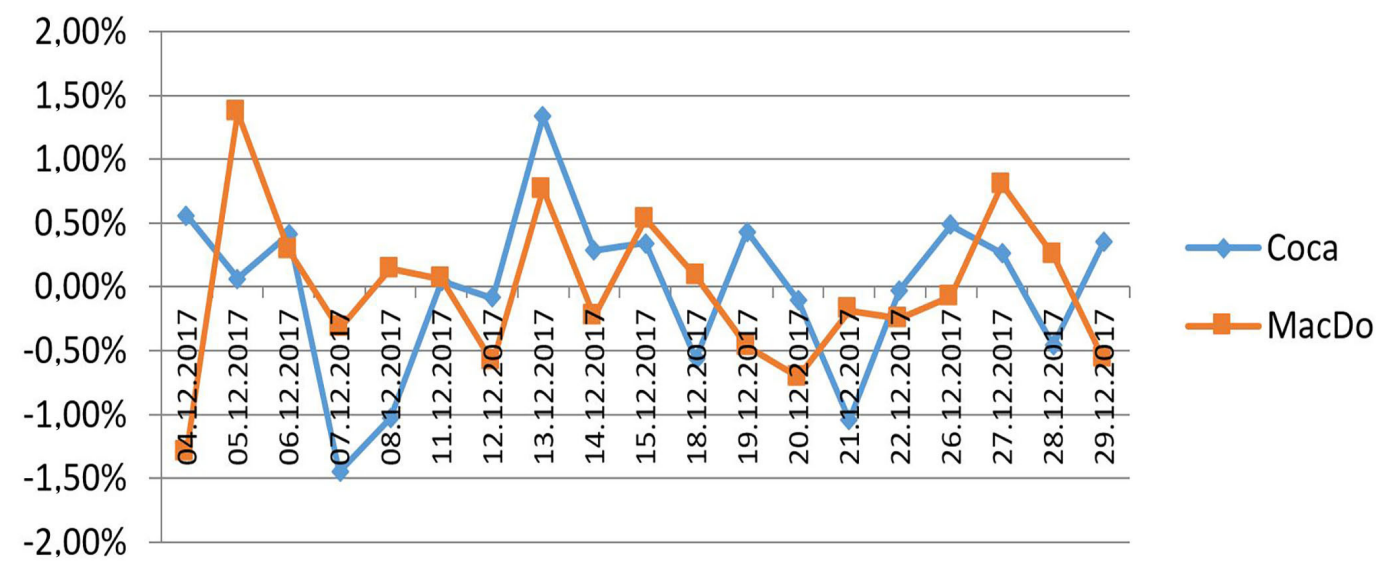

Figura 1. Rentabilidad de Coca Cola y McDonald Fuente: www.investing.com / Elaboración propia

Portafolio 2 - McDonald y Boeing

En este segunda cartera la acción más riesgosa es Boeing, por presentar mayor desviación típica el cual llega a

Tabla 4.
$0,94 \%$, la diferencia con respecto a la primera cartera es que este paquete de activos presenta una correlación negativa, lo cual va a conllevar un riesgo de la cartera del $0,43 \%$, el cual es me- nor que el primer portafolio estructurado. El factor básico para la diferencia de estos resultados es el coeficiente de correlación.

Portafolio de McDonald y Boing / Fuente: www.investing.com / Elaboración propia

\begin{tabular}{ccc} 
& MacDonald & Boing \\
D. Típica & $0,60 \%$ & $0,94 \%$ \\
\hline Cartera & 0,5 & 0,5 \\
\hline Covarianza & $-2,56383 \mathrm{E}-05$ & - \\
\hline C. Correlación & $-45 \%$ & - \\
\hline Varianza & $0,002 \%$ & - \\
\hline D. Típica & $0,43 \%$ & - \\
\hline
\end{tabular}

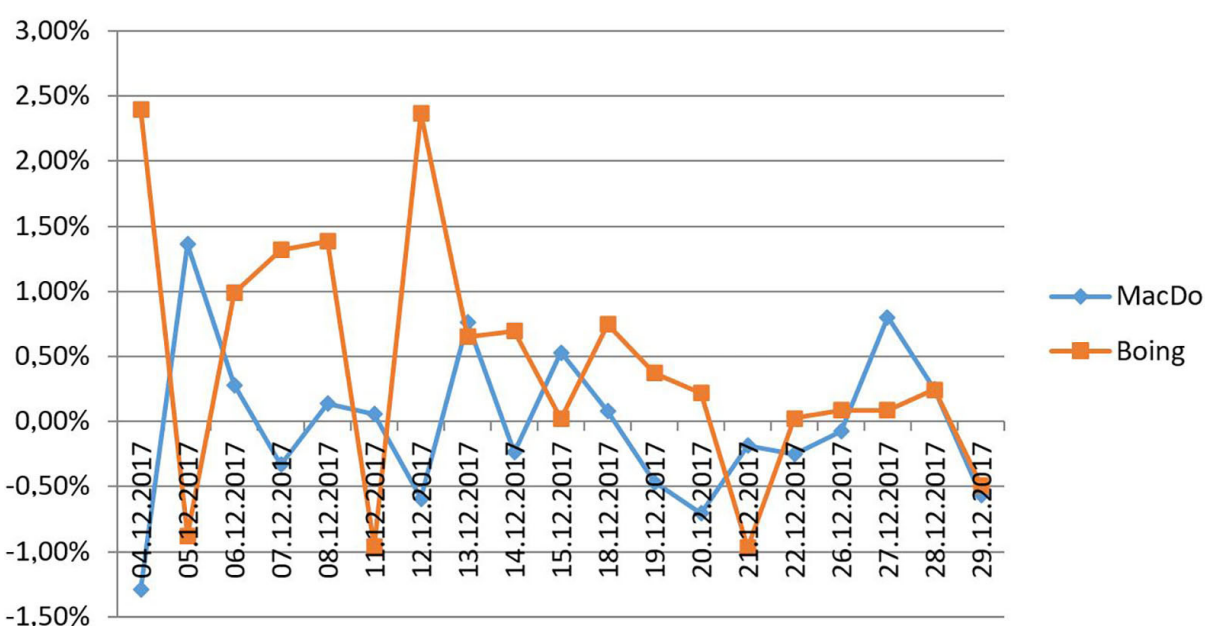

Figura 2. Rentabilidad de McDonald y Boeing Fuente: www.investing.com / Elaboración propia 
La correlación negativa de ambas acciones también se puede observar en el Figura 2, en donde se aprecia el comportamiento asimétrico, pero no perfecto de ambas acciones.
Portafolio 3 - McDonald y Microsoft

De las tres carteras elegidas, esta combinación de activos es la que presenta mayor nivel de riesgo, el cual es explicado por el mayor grado de corre- lación y de tipo positiva que llega al $60 \%$. La volatilidad para esta cartera, medido por la desviación típica es del $0,85 \%$, el cual se podría calificar como de alto riesgo y con débil cobertura.

Tabla 5.

Portafolio de McDonald Y Microsoft / Fuente: www.investing.com / Elaboración propia

\begin{tabular}{ccc} 
& McDonald & Microsoft \\
D. Típica & $0,60 \%$ & $1,28 \%$ \\
\hline Cartera & 0,5 & 0,5 \\
\hline Covarianza & $4,6008 \mathrm{E}-05$ & - \\
\hline C. Correlación & 0,60 & - \\
\hline Varianza & $0,01 \%$ & - \\
\hline D. Típica & $0,85 \%$ & - \\
\hline
\end{tabular}

Como se puede observar, a mayor coeficiente de correlación se obtiene mayor volatilidad de la cartera, el cual queda evidenciado con los resultados obtenidos. Todo lo contrario sucede con la cartera con correlación negativa, como ya se señaló, esta elección va a conducir a menor nivel de riesgo, y se refleja en las desviaciones típicas determinadas para las carteras analizadas.

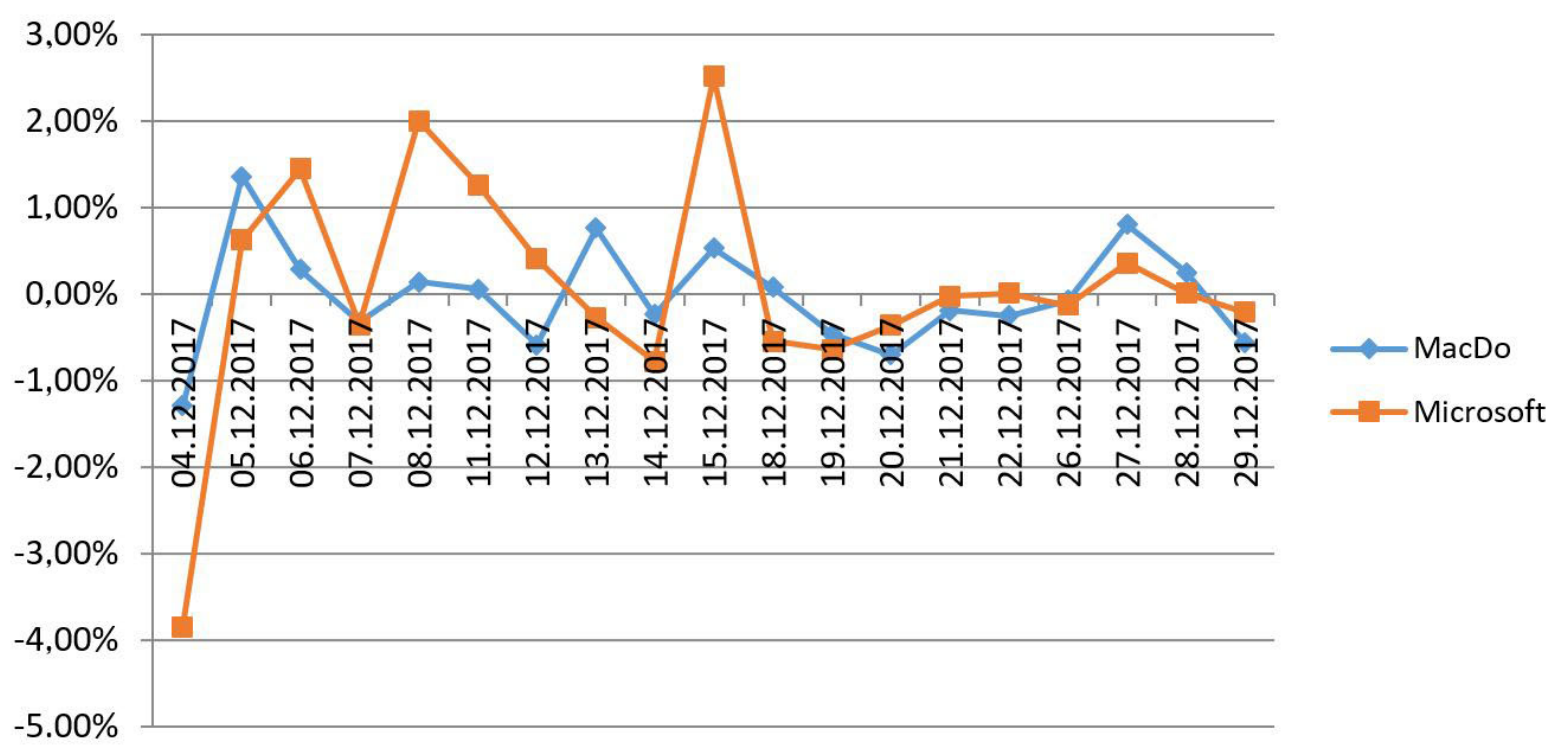

Figura 3. Rentabilidad de McDonald y Microsoft Fuente: www.investing.com / Elaboración propia

En el Figura 3 se nota con claridad la simetría, pero no perfecta del recorrido de la rentabilidad de ambas acciones, que va a conducir a estructurar una cartera de riesgo, en donde la cobertura es un hecho secundario en la toma de decisiones. El objetivo principal es maximizar rentabilidades, pero cabe la probabilidad que el resultado sea lo contrario, es decir se pueda obtener pérdidas importantes, ya que como se señaló, son acciones que se mueven en la misma dirección. Otro indicador básico para tomar decisiones en el mercado bursátil es la 
Beta de las acciones, tal como se planteó en el marco teórico, este indicador, señala como reacciona la rentabilidad de las acciones por cada punto que se mueve el mercado, algunas acciones pueden tener altas o bajas Betas, siendo influenciado en su resultado por el coeficiente de correlación del activo con respecto al mercado $(\mathrm{Rx} / \mathrm{y})$. Cuanto mayor sea este coeficiente, mayor será el Beta del activo, lo cual significa que si un inversionista opta por una acción que está altamente

asociada al comportamiento del mercado, estará asumiendo un alto riesgo, hecho que se evidenciara en un Beta por encima de 1 .

A continuación se corre información de dos acciones enlistadas en sus respectivos Índices Bursátiles. Apple y Caterpillar en el Nadaq y Dow Jones respectivamente.

En cuanto al primer activo, se aprecia una correlación de $47,1 \%$, el cual con- duce a un Beta de 1,38. Con respecto al segundo activo, el coeficiente de correlación llega a 51\%, que conlleva a un Beta de 1,76. Como se puede apreciar en los resultados señalados, dada la volatilidad de los activos y los mercados, el cual es medido por la desviación típica, con un mayor coeficiente de correlación se obtiene un mayor Beta, que en este caso le corresponde a Caterpillar que esta enlistada en el Dow Jones.

Tabla 6.

Rendimiento de Apple y NASDAQ

Fuente: www.investing.com - Elaboración propia

\begin{tabular}{ccc} 
& NASDAQ & APPLE \\
\hline $04 / 12 / 2017$ & $0,24 \%$ & $-0,73 \%$ \\
\hline $05 / 12 / 2017$ & $-0,45 \%$ & $-0,09 \%$ \\
\hline $06 / 12 / 2017$ & $-0,16 \%$ & $-0,37 \%$ \\
\hline $07 / 12 / 2017$ & $0,29 \%$ & $0,18 \%$ \\
\hline $08 / 12 / 2017$ & $0,48 \%$ & $0,03 \%$ \\
\hline $11 / 12 / 2017$ & $0,23 \%$ & $1,93 \%$ \\
\hline $12 / 12 / 2017$ & $0,49 \%$ & $-0,56 \%$ \\
\hline $13 / 12 / 2017$ & $0,33 \%$ & $0,33 \%$ \\
\hline $14 / 12 / 2017$ & $-0,31 \%$ & $-0,03 \%$ \\
\hline $15 / 12 / 2017$ & $0,58 \%$ & $1,01 \%$ \\
\hline $18 / 12 / 2017$ & $0,57 \%$ & $1,40 \%$ \\
\hline $19 / 12 / 2017$ & $-0,15 \%$ & $-1,07 \%$ \\
\hline $20 / 12 / 2017$ & $-0,11 \%$ & $-0,11 \%$ \\
\hline $21 / 12 / 2017$ & $0,22 \%$ & $0,38 \%$ \\
\hline $22 / 12 / 2017$ & $-0,11 \%$ & $0,00 \%$ \\
\hline $26 / 12 / 2017$ & $-0,03 \%$ & $-2,57 \%$ \\
\hline $27 / 12 / 2017$ & $0,11 \%$ & $0,02 \%$ \\
\hline $28 / 12 / 2017$ & $0,25 \%$ & $0,28 \%$ \\
\hline $29 / 12 / 2017$ & $-0,48 \%$ & $-1,09 \%$ \\
\hline
\end{tabular}

Tabla 7.

Resumen estadístico Mercado y Activo

Fuente: www.investing.com - Elaboración propia

\begin{tabular}{ccc} 
& MERCADO & ACTIVO \\
Desviación Típica & 0,003 & 0,009 \\
\hline Correlación & 0,471 & - \\
\hline Beta del activo & 1,38 & - \\
\hline
\end{tabular}




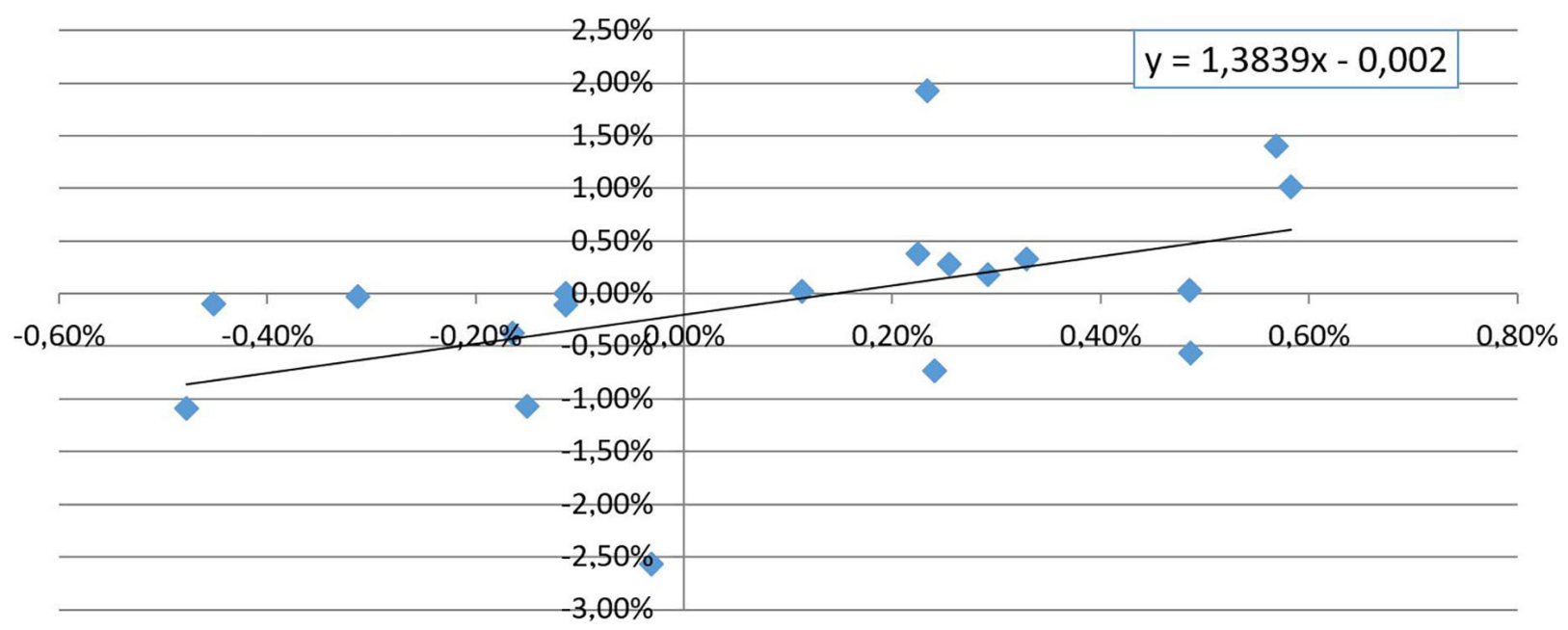

APPLE Lineal (APPLE)

Figura 4. Rendimiento de Apple con respecto al NASDAQ

Fuente: www.investing.com / Elaboración propia

Tabla 8 .

Rendimiento de Caterpillar y Dow Jones

Fuente: www.investing.com - Elaboración propia

\begin{tabular}{ccc} 
& DOW JONES & CATERPILLAR \\
\hline $04 / 12 / 2017$ & 0,0048 & 0,0053 \\
\hline $05 / 12 / 2017$ & $-0,0025$ & $-0,0057$ \\
\hline $06 / 12 / 2017$ & $-0,0011$ & $-0,0069$ \\
\hline $07 / 12 / 2017$ & 0,0003 & $-0,0044$ \\
\hline $08 / 12 / 2017$ & 0,0011 & $-0,0072$ \\
\hline $11 / 12 / 2017$ & $-0,0022$ & $-0,0138$ \\
\hline $12 / 12 / 2017$ & 0,0011 & $-0,0106$ \\
\hline $13 / 12 / 2017$ & 0,0015 & $-0,0076$ \\
\hline $14 / 12 / 2017$ & $-0,0057$ & $-0,0208$ \\
\hline $15 / 12 / 2017$ & $-0,0058$ & $-0,0028$ \\
\hline $18 / 12 / 2017$ & 0,0031 & 0,0155 \\
\hline $19 / 12 / 2017$ & $-0,0033$ & $-0,0353$ \\
\hline $20 / 12 / 2017$ & $-0,0049$ & 0,0024 \\
\hline $21 / 12 / 2017$ & $-0,0023$ & 0,0006 \\
\hline $22 / 12 / 2017$ & $-0,0048$ & $-0,0068$ \\
\hline $26 / 12 / 2017$ & $-0,0029$ & $-0,0180$ \\
\hline $27 / 12 / 2017$ & 0,0016 & $-0,0014$ \\
\hline $28 / 12 / 2017$ & 0,0045 & 0,0097 \\
\hline $29 / 12 / 2017$ & $-0,0024$ & 0,0001 \\
\hline
\end{tabular}


Tabla 9.

Resumen estadístico Mercado y Activo

Fuente: www.investing.com - Elaboración propia

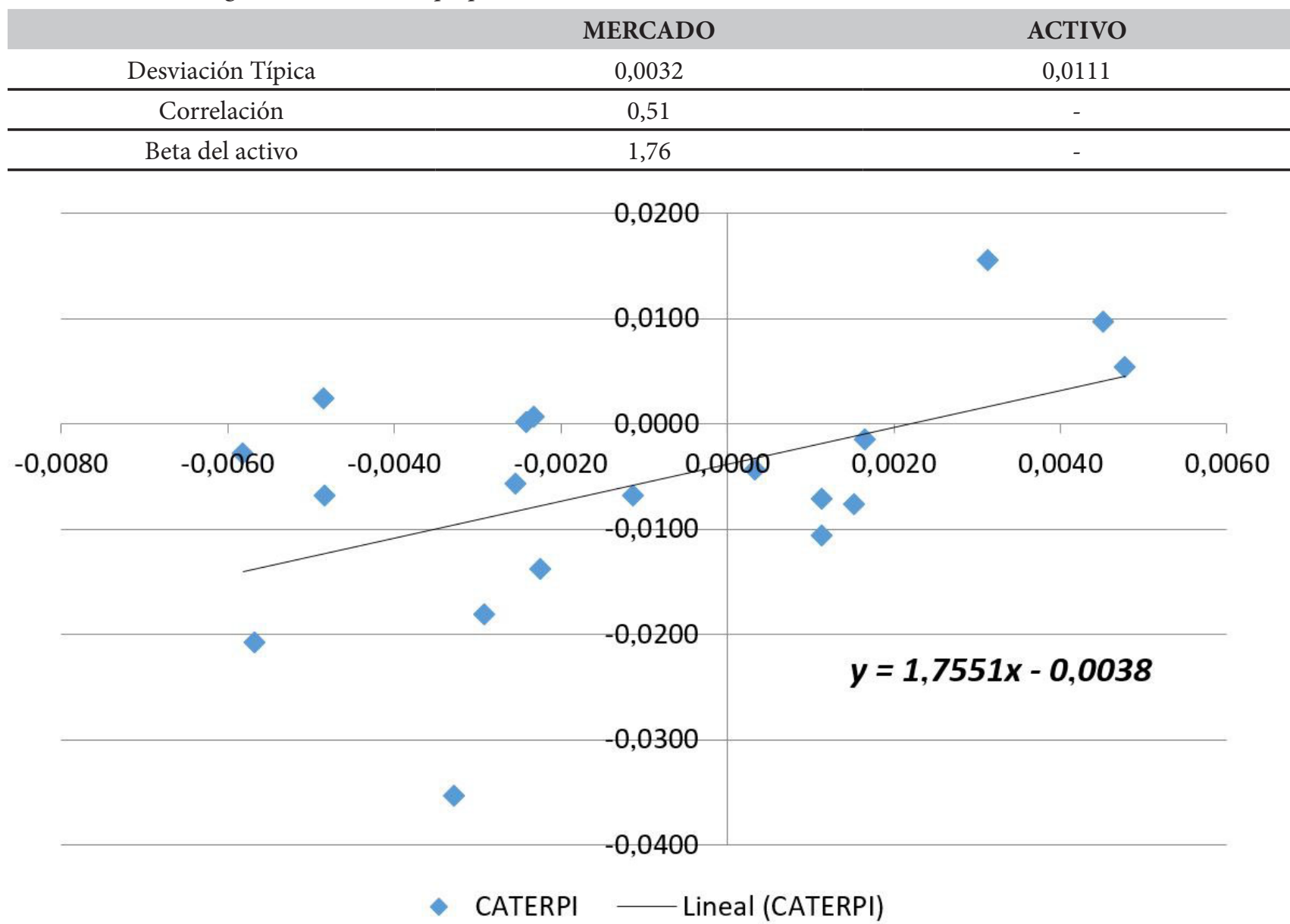

Figura 5. Rendimiento de Caterpillar con respecto al Dow

Jones Fuente: www.investing.com / Elaboración propia

Los Betas también se pueden obtener determinando una Ecuación de Regresión Simple de tipo lineal, en donde la pendiente de la línea refleja el resultado de este indicador financiero. Siendo la Beta de Apple de 1,38 y de Caterpillar de 1,76. Ambos activos se pueden calificar como riesgosos o agresivos, en especial Caterpillar que alcanza el mayor Beta. Estos resultados son exactamente igual a lo obtenido por el método en donde se utilizó el coeficiente de correlación y la desviación típica del mercado y de los activos.

\section{CONCLUSIONES}

Para estructurar portafolios de activos financieros de renta variable, como son las acciones, es conveniente tomar en cuenta el coeficiente de correlación de los activos elegidos, ya que en función del resultado, se podrá determinar si son carteras de alto o bajo riesgo.

Cuanto más negativo sea el coeficiente de correlación de los activos, mayor será la cobertura ante los riesgos por la estructuración de portafolios de activos financieros de renta variable. La cobertura seria perfecta si el coeficiente de correlación es -1. En función de este principio, un inversionista totalmente adverso al riesgo optará por este tipo de cartera.

Cuanto mayor sea el coeficiente de correlación de los activos financieros, mayor será el riesgo que se asume por una cartera se este tipo. Si el coeficiente de correlación es 1 , el inversionista estará totalmente expuesto al riesgo, el cual además se verá reflejada en la elevada desviación típica del portafolio. 
Los betas de los activos de renta variable son influenciados por el coeficiente de correlación. En este caso el estadístico refleja el grado y tipo de asociatividad del rendimiento del activo con respecto al mercado. Cuanto mayor sea esta relación mayor será el beta del activo, con lo cual el grado de sensibilidad de la acción con respecto al mercado será mayor, por lo que se podría tipificar al activo como de alto riesgo.

Es un estudio que amerita seguir profundizando para desarrollar instrumentos de decisión en el mercado bursátil y así los inversionistas puedan optimizar sus decisiones.

\section{REFERENCIAS BIBLIOGRÁFICAS}

Brealey, R., Myers, S. \& Marcus, A. (2004). Fundamentos de finanzas corporativas. Madrid: McGraw-Hill.

Bodie, Z., Kane, A. \& Marcus A. (2004). Principio de inversiones. Madrid: McGraw-Hill.

Burton, M. (2012). Un paseo aleatorio por Wall Street. Madrid: Alianza Editorial.
Gitman, L. \& Joehnk, M. (2009). Fundamento de inversiones. México: Ediciones Pearson.

Marcelo, E. (2015). Administración de carteras de inversiones. Buenos Aires: Ediciones Macchi.

Serrano, J. (2013). El inversor intranquilo. Madrid: Díaz Santos.

www.investing.com

www.finanzas.com 